\title{
Inversion of borehole-response test data for estimation of subglacial hydraulic properties
}

\author{
Dan B. Stone, ${ }^{1}$ Garry K. C. Clarke, ${ }^{2}$ Robert G. Ellis ${ }^{2}$ \\ ${ }^{1}$ Institute of Arctic and Alpine Research, University of Colorado, Boulder, Colorado 80309-0450, U.S.A. \\ ${ }^{2}$ Department of Geophysics and Astronomy, University of British Columbia, Vancouver, British Columbia V6T 1Z4, Canada
}

\begin{abstract}
Response tests are widely used in ground-water studies to assess the hydraulic properties of sub-surface water-flow systems. The simplicity of such tests also makes them attractive for investigation of subglacial hydraulic conditions. This paper describes a systematic, quantitative approach to the analysis of borehole-response test data. The approach uses the theoretical model of Stone and Clarke (1993), which describes water motion in a coupled borehole-subglacial flow system; this framework provides the basis for an inversion scheme that is focused on quantifying physical properties of the basal-flow system, as it is characterized in the theoretical model. The inversion procedure was applied to response-test data from Trapridge Glacier, Yukon Territory, Canada. Results of the inversions suggest that the subglacial drainage network can be described as a confined layer comprising coarse-sand- to fine-gravel-sized sediments, having a thickness of $0.1-0.3 \mathrm{~m}$, and a hydraulic conductivity of about $5 \times 10^{-4} \mathrm{~m} \mathrm{~s}^{-1}$. Based on the waterdrainage rates from boreholes, as they connect with the subglacial water-flow system, specific storage of the sediment layer was estimated to be approximately $1 \times 10^{-4} \mathrm{~m}^{-1}$. Further consideration of subglacial water-flow conditions suggests that connectiondrainage test results may tend to underestimate specific storage of the overall glacier substrate.
\end{abstract}

\section{NOTATION}

$b \quad$ Thickness of subglacial aquifer $(\mathrm{m})$

$B \quad$ Kinetic energy-loss weighting factor

$d_{i} \quad$ Predicted datum

$d_{i}^{\text {obs }}$ Observed datum

$\delta d_{i}^{\text {obs }}$ Estimated uncertainty in observed datum

E Total data misfit

$E_{\%} \quad$ Percentage misfit

$g \quad$ Gravitational acceleration $\left(\mathrm{m} \mathrm{s}^{-2}\right)$

$h_{0} \quad$ Initial hydraulic head $(\mathrm{m})$

$h_{\mathrm{i}} \quad$ Thickness of glacier ice $(\mathrm{m})$

$h^{\star} \quad$ Dimensionless water-column height

$h_{\mathrm{B}}^{\star} \quad$ Dimensionless hydraulic head

$k \quad$ Intrinsic permeability $\left(\mathrm{m}^{2}\right)$

$K \quad$ Hydraulic conductivity $\left(\mathrm{m} \mathrm{s}^{-1}\right)$

$m_{i} \quad$ Model parameter

$m_{i}^{\text {est }} \quad$ Estimated model parameter value

$\delta m_{i}^{\text {est }}$ Estimated uncertainty in model parameter value

$M \quad$ Number of model parameters

$n$ Porosity

$N \quad$ Number of data
Dimensionless specific discharge

Filter, inner boundary radius ( $\mathrm{m}$ )

Outer boundary radius ( $\mathrm{m}$ )

Well, borehole radius ( $\mathrm{m}$ )

Dimensionless radial coordinate

Critical Reynolds number

Solid surface-to-volume ratio $\left(\mathrm{m}^{-1}\right)$

Specific storage $\left(\mathrm{m}^{-1}\right)$

Dimensionless time

Transmissivity $\left(\mathrm{m}^{2} \mathrm{~s}^{-1}\right)$

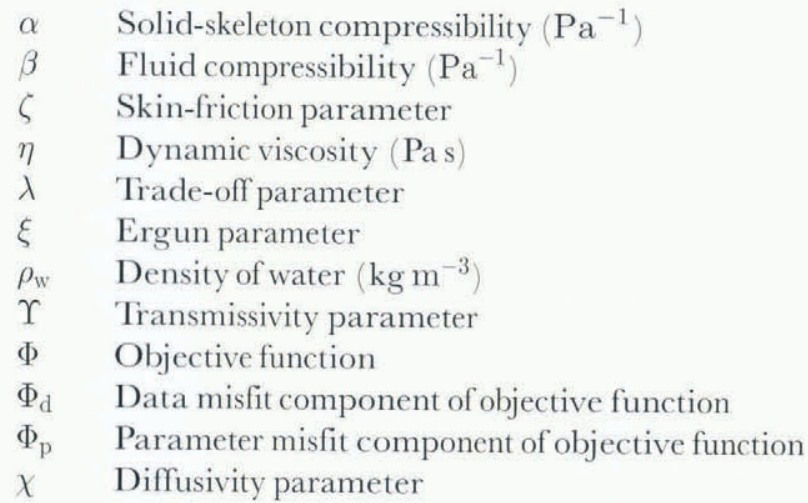

\section{INTRODUCTION}

In a previous paper, Stone and Clarke (1993) developed a theoretical framework for borehole-response tests (BHRT) on glaciers and demonstrated how their model could be used to solve the forward problem - generating predicted data which can be compared with field observations. In this paper, we consider the inverse problem, which begins with the observed data and is guided by the forward model toward an estimate of the model parameters. Forward modeling describes the underlying physics of a particular system; inversion uses the modeled or assumed physics and focuses on quantifying specific properties of the system.

The BHRT model represents subglacial water-flow conditions in an idealized way. The model describes water flow in a saturated, confined sediment layer at the glacier sole. It is in this context that we will use the terms "aquifer" and "flow layer" when referring to the subglacial drainage system. The model also assumes that subglacial material in 
the region influenced by a response test is homogeneous and isotropic. This approach avoids the complications of a more detailed description and, instead, represents the actual substrate in terms of an effective medium whose hydraulic properties incorporate the test-scale heterogeneities; the effective medium is an alternate, but hydraulically equivalent, characterization of the real flow system.

Data from two different types of response tests will be examined: the first we refer to as a connection-drainage test, which entails monitoring the water-level draw-down with time when a water-filled borehole is suddenly opened to the subglacial aquifer; the second is a conventional slug test, in which a sealed pipe is suddenly removed from an equilibrated borehole and the water-level recovery with time is measured. Both types of test have previously been used to investigate subglacial hydraulic conditions (Hodge, 1976; Engelhardt, 1978; Engelhardt and others, 1990; Fountain, 1994; Iken and others, 1996). This paper describes a systematic, quantitative approach to the analysis of such data sets, which has until now been lacking in glaciological studies.

The BHRT framework of Stone and Clarke departs from standard ground-water models (e.g. Cooper and others, 1965; Kamp, 1976; Kipp, 1985), which are based on Darcy's law and the assumption that water velocities are low; under the highgradient conditions that arise during connection-drainage tests on glaciers, Reynolds numbers may be large and turbulent effects can play an important role. The BHRT model provides for a continuous transition between laminar- and turbulent-flow regimes and reduces to Darcy's law at low Reynolds numbers.

Besides turbulent effects during a connection-drainage test, response testing on glaciers has additional complexity because the time-scale over which hydraulic head naturally varies in the subglacial environment can be similar to the time-scale of a response test. Determining the change in background head during a response test and accounting for this effect is an important step in the analysis of glacier response-test data. We describe our approach to this problem in the following section.

This paper contains only a brief statement of the forward model; the reader is referred to Stone and Clarke (1993) for a comprehensive discussion of the BHRT model and its numerical method of solution. Here, we describe a systematic method for analyzing BHRT data and demonstrate its application to field data. The procedure was used to invert response-test data collected on Trapridge Glacier $\left(61^{\circ} 14^{\prime} \mathrm{N}, 140^{\circ} 20^{\prime} \mathrm{W}\right)$, a small sub-polar glacier in Yukon Territory, Canada. We show results from inversions of eight data sets and describe how physical properties of the flow system are extracted from the final estimates of model parameters. We conclude by comparing our inversion results with reported estimates of subglacial hydraulic properties obtained by other means, both on Trapridge Glacier and elsewhere.

\section{METHODOLOGY}

\section{Data collection and processing}

We used pressure sensors suspended in boreholes to monitor water levels during response tests. Readings of borehole water pressure were collected every $0.0625 \mathrm{~s}$ during disturbances, while background trends and long-term recoveries were recorded at $2.0 \mathrm{~s}$ intervals. To facilitate comparisons with model solutions, each data set was fitted with an interpolating spline (Press and others, 1989, p. 88) and data having a uniform spacing of $0.125 \mathrm{~s}$ were generated from it. For connection-drainage tests, a constant offset was added to the data so that readings prior to connection correspond to a water-column height equal to the glacier thickness. For slug tests, the data were shifted so that water-pressure readings were converted to displacements relative to a pre-disturbance datum.

Under "typical" ground-water conditions, the hydraulic head at a particular point within an aquifer usually changes slowly with time. Over the relatively short time span of a response test it is, therefore, reasonable to assume that the disturbance is a brief perturbation to a constant background head. For a subglacial aquifer, however, this assumption can be untenable: glacier motion and diurnal cycles of meltwater input and discharge cause continual and sometimes large changes in hydraulic head at a given location. (A response test might be performed, for instance, during a rapidly rising limb in basal water pressure resulting from a period of enhanced meltwater production.) Such effects must either be modeled or be removed from the data before response-test results can be analyzed. Our approach is to remove background trends from the data whenever they can be determined.

Background trends in basal water pressure can be estimated from measurements of borehole water-level variations prior to a disturbance. Obviously, this can only be done after the borehole has been drilled, or if there are other communicating boreholes nearby. For the drainage tests considered in this paper, we did not have suitable boreholes nearby, and so are unable to determine background trends in subglacial water pressure at the times of these disturbances. However, since these connections took place rapidly - approaching equilibrium in about $1 \mathrm{~min}$ or less - the change in background pressure over such short time intervals is probably negligible, so results from these tests should still be valid. For slower drainage rates following a connection, this would not be true.

Borehole water levels were recorded before slug tests, and we apply this information to the data prior to inversion. Our procedure is illustrated in Figure 1 and is explained here. Data prior to initial disturbances, shown as dotted segments in Figure la and c, are fitted with straight lines. These straight-line fits approximate the background trends in basal water pressure at the times that the tests were performed. The trends are removed by subtracting the fitted straight lines from the entire data series, resulting in the adjustments shown in Figure $\mathrm{lb}$ and $\mathrm{d}$. The horizontal dashed lines in Figure $\mathrm{lb}$ and $\mathrm{d}$ indicate datums, which are defined by the pre-disturbance water levels following removal of background trends.

\section{Forward model}

The model of Stone and Clarke (1993) describes water flow in a borehole subglacial aquifer system during a response test. In simplified form, the BHRT model is expressed by the following dimensionless equations: 

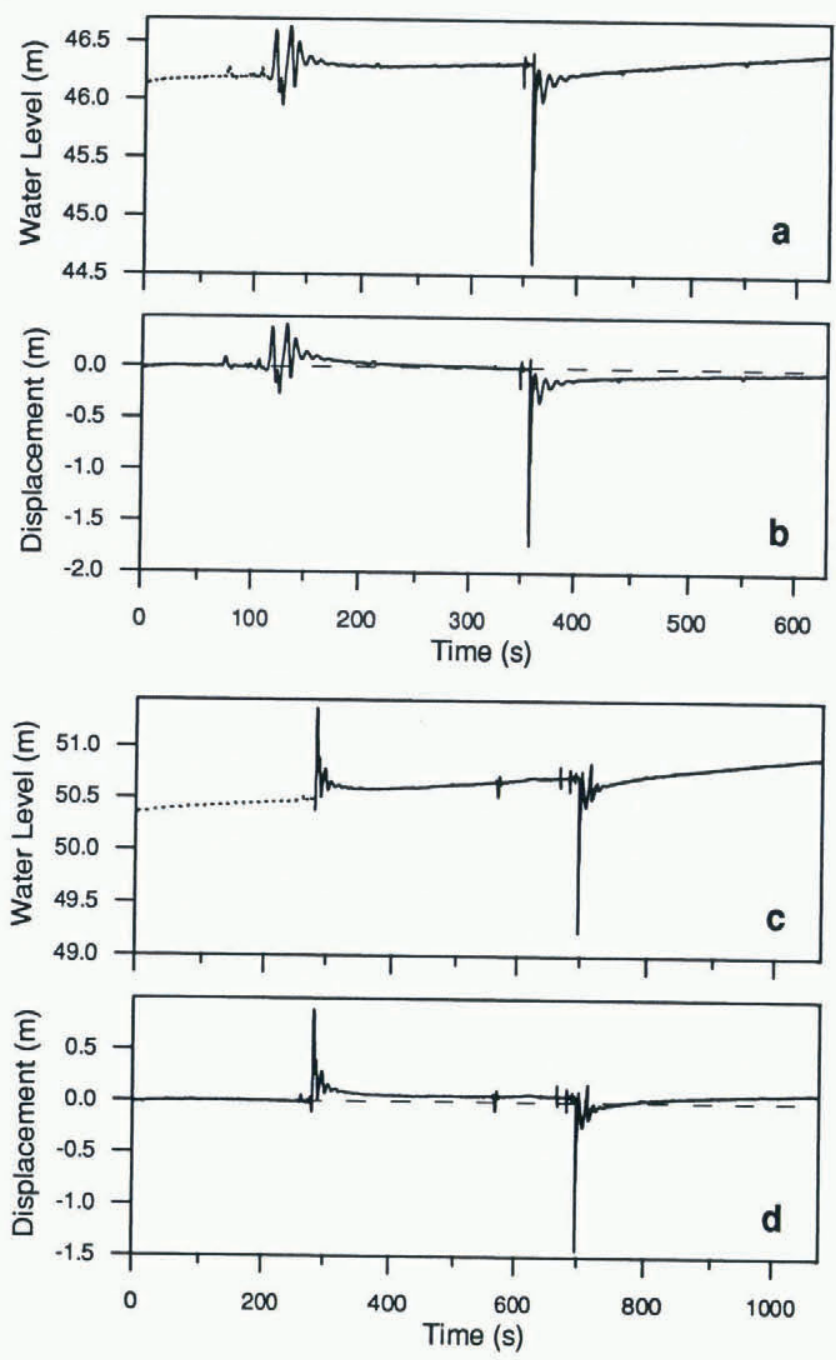

Fig. 1. Removal of background trends illustrated for two slug tests: (a) 90ST38A and (c) 90ST38E. The procedure is explained in the text. (b) For 90ST38A, the system was fully re-equilibrated at the time the slug was removed. (d) In the case of 90ST38E, the slug was removed when the water level was higher than its pre-disturbed value.

$$
\begin{aligned}
& h^{\star} \frac{\mathrm{d}^{2} h^{\star}}{\mathrm{d} t^{\star 2}}+\zeta h^{\star} \frac{\mathrm{d} h^{\star}}{\mathrm{d} t^{\star}}+h^{\star}=h_{\mathrm{B}}^{\star}\left(1, t^{\star}\right), \\
&-\frac{1}{r^{\star}} \frac{\partial}{\partial r^{\star}}\left(r^{\star} q^{\star}\right)=\frac{1}{\chi} \frac{\partial h_{\mathrm{B}}^{\star}}{\partial t^{\star}}, \\
& q^{\star}\left(1, t^{\star}\right)=-\frac{1}{\Upsilon} \frac{\mathrm{d} h^{\star}}{\mathrm{d} t^{\star}}, \\
& q^{\star}=-(2) \frac{\partial h_{\mathrm{B}}^{\star}}{\partial r^{\star}}\left[1+\left(1+\xi\left|\frac{\partial h_{\mathrm{B}}^{\star}}{\partial r^{\star}}\right|\right)^{\frac{1}{2}}\right]^{-1}
\end{aligned}
$$

(Stone and Clarke, 1993, equations (35)-(38)). Motion of water in the borehole is described by Equation (1), while Equation (2) governs radial water flow in the subglacial aquifer. Transfer of water between the borehole and the aquifer is expressed by Equation (3). The constitutive relation in Equation (4), which could be substituted into Equations (2) and (3), has been explicitly stated to show the allowance for non-Darcian behavior.

Equations (1)-(4) contain four dimensionless parameters: the borehole skin-friction parameter $\zeta$, the diffusivity parameter $\chi$, the transmissivity parameter $\Upsilon$ and the "Ergun" parameter $\xi$. In terms of actual physical quantities, these parameters are defined as follows:

$$
\begin{aligned}
\zeta & =\left(\frac{8 \eta}{\rho_{\mathrm{w}} r_{\mathrm{w}}^{2}}\right)\left(\frac{h_{0}}{g}\right)^{\frac{1}{2}}, \\
\chi & =\left(\frac{K}{S_{\mathrm{s}} r_{\mathrm{f}}^{2}}\right)\left(\frac{h_{0}}{g}\right)^{\frac{1}{2}}, \\
\Upsilon & =\left(\frac{2 K b}{r_{\mathrm{w}}^{2}}\right)\left(\frac{h_{0}}{g}\right)^{\frac{1}{2}}, \\
\xi & =\left(\frac{K^{2} B S_{0}(1-n) h_{0}}{2 r_{\mathrm{f}} g n^{3}}\right) .
\end{aligned}
$$

The hydraulic properties in these expressions that are of primary interest are the hydraulic conductivity $K$, the specific storage $S_{\mathrm{s}}$ and the flow-layer thickness $b$. The quantity $B$ in Equation (8a) is a coefficient that determines the partitioning of terms in Equation (4) under conditions of laminar or turbulent flow. As discussed by Stone and Clarke (1993, p. 332), $B$ can be expressed in terms of the porosity $n$ and a critical Reynolds number Re', which is the Reynolds number corresponding to the transition between laminarand turbulent-flow regimes: $B=240(1-n) / \operatorname{Re}^{\prime}$. Substituting this expression into Equation (8a) yields

$$
\xi=\left(\frac{120 K^{2} S_{0}(1-n)^{2} h_{0}}{r_{\mathrm{f}} g n^{3} \operatorname{Re}^{\prime}}\right) .
$$

The boundary and initial conditions for the forward model have been described by Stone and Clarke (1993, p. 333). As discussed in that paper, the model allows for either a constant-head or a no-flux outer-boundary condition. For the inversions presented in this paper, we have used a constant-head outer-boundary condition. Thus, we assume that at a sufficient distance from the borehole the pre-existing hydraulic head in the aquifer is unaffected by a response-test disturbance; this distance corresponds to the outer boundary radius.

\section{Inversion scheme}

The forward model represents a non-linear functional relationship between the model parameters $m_{i}$ and the predicted borehole water-level data $d_{i}$. If the model accurately characterizes the geometry and physics of the subglacial water-flow system and if the initial conditions and model parameters are well known, then $d_{i}$ will closely approximate observed response-test data $d_{i}^{\text {obs }}$. To gauge the accuracy of the model and how well its inputs are known, a measure of the discrepancy between predicted and observed data is required. Our inversion scheme utilizes the following objective function for that purpose:

$$
\Phi=\Phi_{\mathrm{d}}+\lambda \Phi_{\mathrm{p}}
$$

where $\Phi_{\mathrm{d}}$ is a measure of the data misfit, $\Phi_{\mathrm{p}}$ is a measure of the misfit between initial "best-guess" model parameters and the current values for $m_{i}$, and $\lambda$ is an adjustable constant that determines the relative importance of the parameter and data-misfit terms. A detailed explanation of Equation (9) is given in the Appendix. The influence of $\lambda$ on the final value of $\Phi$ is discussed below.

To minimize the objective function in Equation (9), inversion parameters are adjusted using a quasi-Newton method. The inversion algorithm that we use is a double precision optimization routine given by Kahaner and others (1989, p. 372). We adjusted the accuracy level of the inversion algorithm to mesh with the forward model- 
integration scheme; this was necessary to ensure that changes in inversion parameters, which are made by the inversion routine, produce numerically distinguishable changes in the forward model solution.

To evaluate the relative success of different models, we calculate a percentage misfit $E_{\%}$, defined as

$$
E_{\%}=100 \times \frac{1}{N} \sum_{i=1}^{N}\left|\frac{d_{i}^{\text {obs }}-d_{i}}{d_{\max }^{\text {obs }}-d_{\min }^{\text {obs }}}\right| .
$$

The quantity $E_{\%}$ indicates the relative accuracy of a given set of predicted data; smaller percentage misfits are associated with more accurately predicted data.

\section{Inversion parameters and model inputs}

If the solution is to be physically plausible, all four dimensionless parameters must be non-negative. To enforce nonnegativity of model parameters, we distinguish between inversion and model parameters, and we define the inversion parameters as the natural logarithms of the model parameters: hence, the inversion parameters are $m_{1}=\ln \zeta$, $m_{2}=\ln \chi, m_{3}=\ln \Upsilon$ and $m_{4}=\ln \xi$. This scheme requires that antilogarithms of the inversion parameters be taken before computing forward-model solutions.

For inversions of connection-drainage data, we include a fifth parameter that specifies the time of the connection. In general, this quantity is not accurately known and it can significantly influence inversion results. By including the time of connection as an inversion parameter, we obtain an optimal estimate of when the borehole started to drain. However, because this quantity is not of hydrologic interest, we exclude it from subsequent discussions.

The uncertainties that we associate with the inversion parameters are based on plausible upper and lower limits for the dimensionless model parameters. We have selected plausible limits for $\zeta$ on physical grounds by considering only realistic values for the borehole radius. Plausible bounds for the other parameters were chosen based on reasonable extremes in forward-modeling results. The difference between the logarithms of the limits assigned to the plausible model parameters is the estimated uncertainty that we associate with a given inversion parameter: $\delta m_{i}^{\text {est }}=$ $m_{i}^{\text {upper }}-m_{i}^{\text {lower }}$, where, for example, $m_{2}^{\text {upper }}=\ln \chi^{\text {upper }}$. With this definition, better-constrained parameters, such as $\zeta$, are associated with a smaller uncertainty.

The forward model requires input of quantities other than the four dimensionless parameters. Material constants such as the density, viscosity and compressibility of water must be specified, as well as geometric quantities like the ice thickness and the aquifer inner and outer boundary radii. Values for these model inputs, which were held constant for all inversions, are listed in Table 1.

In addition to the inputs listed in Table 1, the model also requires specification of the initial hydraulic head $h_{0}$ at the location and time that a particular response test was performed. This quantity is not a constant and must be known or estimated for each test. Table 3 lists the values of $h_{0}$, determined from field measurements, that were used in the inversions.

\section{Inversion procedure}

The inversion algorithm requires (1) a way of solving the forward problem, (2) a measure of model success in fitting
Table 1. Model inputs held constant for inversions

\begin{tabular}{lccc}
\hline Model input & Symbol & Value & Units \\
& & & \\
\hline & & & \\
Water density & $\rho_{\mathrm{w}}$ & $1.0 \times 10^{3}$ & $\mathrm{~kg} \mathrm{~m}^{-3}$ \\
Water viscosity & $\beta$ & $1.787 \times 10^{-3}$ & $\mathrm{~Pa} \mathrm{~s}^{-10}$ \\
Water compressibility & $h_{\mathrm{i}}$ & 70.0 & $\mathrm{~Pa}^{-1}$ \\
Ice thickness & $r_{\mathrm{f}}$ & 0.05 & $\mathrm{~m}$ \\
Inner boundary radius & $r_{\text {max }}$ & 200.0 & $\mathrm{~m}$ \\
Outer boundary radius & $\delta d_{i}^{\text {obs }}$ & 0.05 & $\mathrm{~m}$ \\
Uncertainty in data & $\delta m_{1}^{\text {est }}$ & 2.3 & $\mathrm{~m}$ \\
Uncertainty in $\ln \zeta^{\text {est }}$ & $\delta m_{2}^{\text {est }}$ & 18.4 & \\
Uncertainty in $\ln \chi^{\text {est }}$ & $\delta m_{3}^{\text {est }}$ & 8.3 & \\
Uncertainty in $\ln \Upsilon^{\text {est }}$ & $\delta m_{4}^{\text {est }}$ & 9.2 & \\
Uncertainty in $\ln \xi^{\text {est }}$ & $\lambda$ & 0.01 & \\
Trade-off parameter & & & \\
& & & \\
\hline
\end{tabular}

the observed data and (3) initial estimates of model parameters. The BHRT model satisfies the first requirement and the second is provided by the objective function. The third requirement - providing initial estimates of model parameters - is handled using an iterative procedure, the details of which are described in Appendix B.

The iterative inversion procedure is illustrated in Figure 2 using data from slug test 90ST38A. (Individual response tests are distinguished using an alpha-numeric label: the first two numbers indicate the year that the test was performed, the following two-character string identifies the type of test, the next two numbers denote the borehole and the last character shows the sequential position in a series of

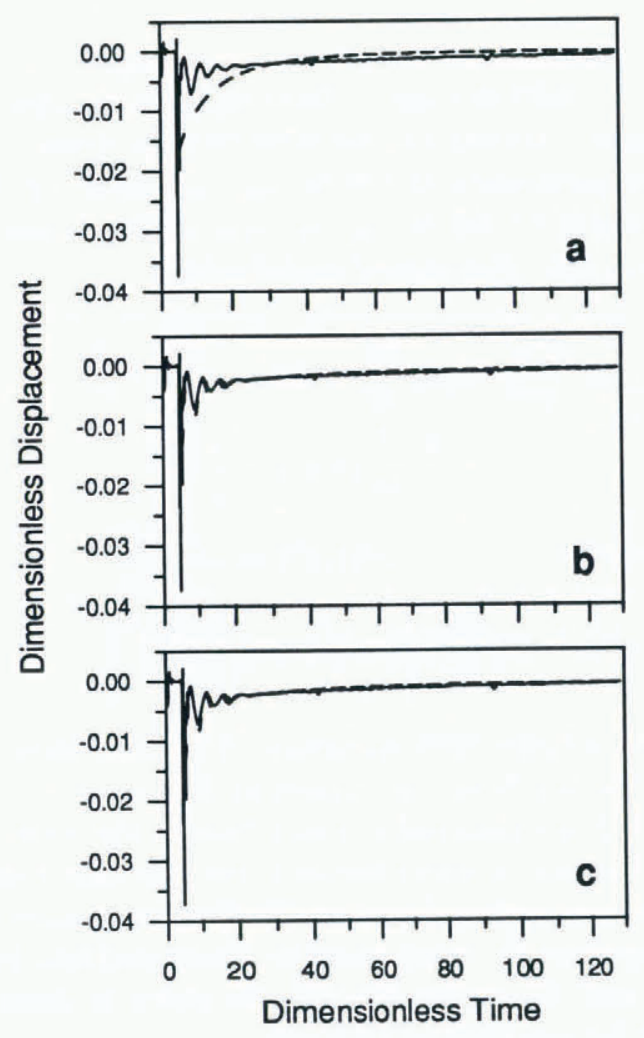

Fig. 2. Iterative inversion procedure illustrated with data from slug test 90ST38A (solid lines) and modeled results (dashed lines). Model input parameters are listed in Table 2. The procedure is explained in Appendix B. 
slug tests.) Initial model-parameter estimates and associated values of $\Phi$ and $E_{\%}$ are listed in Table 2.

The dashed line in Figure $2 \mathrm{a}$ is the forward model solution based on our initial parameter estimates. For this test, we began by trying to fit only the long-term trend, not the initial oscillations. The final set of parameters obtained by the first inversion gave the solution shown in Figure 2b; these were then used as the starting values for a second inversion. As can be seen in Figure 2b, after the first inversion the fit to the observed data was noticeably improved. Values of $\Phi$ and $E_{\%}$ following the second inversion were only slightly different from the previous ones. In this particular case, we performed three additional inversions, resulting in the final solution shown in Figure 2c.

\section{Influence of trade-off parameter}

By virtue of the parameter misfit term in the objective function in Equation (9), an optimal solution - the set of model parameters that results in the smallest value of the objective function - will not necessarily correspond to the smallest total data misfit. The non-linear nature of the forward model and its numerical method of solution forces a tradeoff between fitting the observed data and stabilizing the search procedure. We have examined the degree to which the trade-off parameter $\lambda$ in Equation (9) influences the final value of $\Phi$; our analysis is summarized in this section.

The influence of the trade-off parameter $\lambda$ on the final values of $\Phi_{\mathrm{d}}$ and $\Phi_{\mathrm{p}}$ was assessed by varying only $\lambda$ in repeated inversions of data from one response test (90ST38A); we performed 10 inversions, changing $\lambda$ by one order of magnitude each time. The initial model-parameter estimates for these inversions were the same as those listed for Figure 2a in Table 2. We point out that for $\lambda=0.01$ an inversion started with these initial parameter estimates produced the final set of parameters listed for Figure $2 \mathrm{~b}$ in Table 2, and that the final parameters obtained in this inversion were substantially different from the initial estimates. Thus, this particular inversion approximates a worst-case scenario: the total parameter misfit is large, so the influence of $\lambda$ should be strongly felt.

Final objective-function values resulting from these inversions were used to generate relationships between $\lambda$ and the two misfit terms $\Phi_{\mathrm{d}}$ and $\Phi_{\mathrm{p}}$ (Fig. 3). These relationships show that the data misfit is virtually constant for $\lambda \leq 1$, while it changes rapidly for $\lambda>10$. Also, the parameter misfit is gradually reduced as $\lambda$ is increased, because the requirement of closely matching initial parameter estimates becomes dominant. It is apparent that for $\lambda \leq 10^{-2}$ the parameter misfit term does not significantly contribute

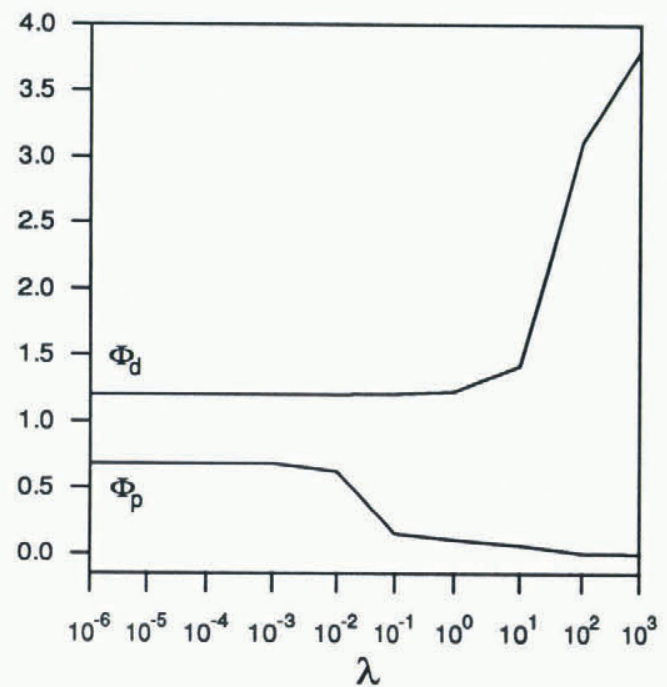

Fig. 3. Trade-off curve based on inversions of slug test 90ST 38A data: size of data $\Phi_{\mathrm{d}}$ and parameter $\Phi_{\mathrm{p}}$ misfit terms as a function of trade-off parameter $\lambda$

to the final value of $\Phi$, since the data misfit is large relative to $\lambda \Phi_{\mathrm{p}}$. On the other hand, for $\lambda \geq 1$, the parameter-misfit term forces an appreciable increase in $\Phi_{\mathrm{d}}$ and moves the solution away from the desired goal of fitting the data. Therefore, based on this analysis, we have used a constant value of $\lambda=0.01$ for all inversions in this study.

\section{RESULTS}

Inversion results are presented for eight response tests, four of each type of test, which were performed on Trapridge Glacier in the summer of 1990. The four connection-drainages were recorded in boreholes in close proximity - the maximum separation distance was $34.9 \mathrm{~m}$ (holes 38 and 59) and the minimum distance was $5.3 \mathrm{~m}$ (holes 23 and 38). Three of the four slug tests were performed in hole 38 in a single day - the first at $1448 \mathrm{~h}$ (90ST38A), the second at $1654 \mathrm{~h}$ (90ST38E) and the third at $1730 \mathrm{~h}(90 \mathrm{ST} 38 \mathrm{G})$. The starting points for inversions of these data sets are given in Table 3.

Estimated model parameters obtained by the inversions were used to generate final sets of predicted data. The final parameter estimates are listed in Table 4. Comparisons of predicted and observed data are shown in Figure 4, where we have re-dimensionalized the predicted data and shown them with dashed lines.

In general, the correlations between observed data and the predicted final models are excellent; even when the finer

Table 2. Parameter estimates, objective function, and data misfit values for iterative inversions of slug test 90ST38A, as shown in Figure 2

\begin{tabular}{|c|c|c|c|c|}
\hline & & $m^{\text {est }}$ & & $m^{\text {final }}$ \\
\hline Iteration & 0 & 1 & 2 & 5 \\
\hline Figure & a & $\mathrm{b}$ & (not shown) & c \\
\hline$\zeta$ & $1.000 \times 10^{-2}$ & $2.765 \times 10^{-3}$ & $2.764 \times 10^{-3}$ & $2.763 \times 10^{-3}$ \\
\hline$x$ & $1.000 \times 10^{+3}$ & $3.758 \times 10^{-2}$ & $3.683 \times 10^{-2}$ & $3.622 \times 10^{-2}$ \\
\hline$\hat{\Upsilon}$ & $5.000 \times 10^{-1}$ & $1.088 \times 10^{-1}$ & $1.070 \times 10^{-1}$ & $1.066 \times 10^{-1}$ \\
\hline$\xi$ & $1.000 \times 10^{+3}$ & $7.793 \times 10^{-4}$ & $7.793 \times 10^{-4}$ & $7.793 \times 10^{4}$ \\
\hline$\Phi$ & 5.093 & 1.208 & 1.201 & 1.200 \\
\hline$E_{\%}$ & 2.01 & 1.07 & 1.02 & 1.02 \\
\hline
\end{tabular}


Table 3. Initial estimates of model parameters and hydraulic head for inversions shown in Figure 4

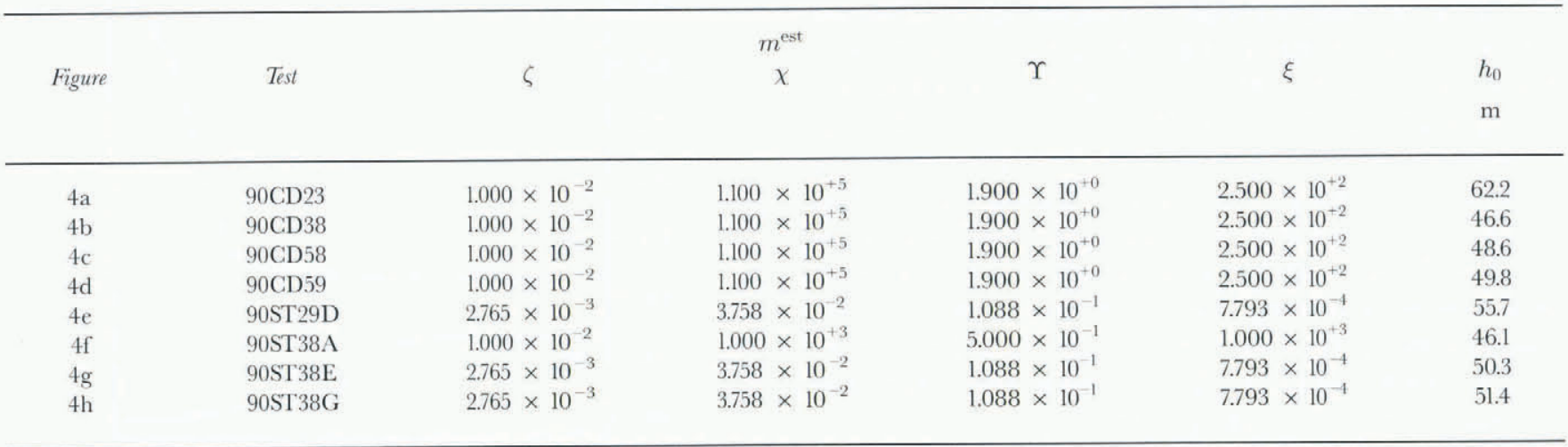

Table 4. Final estimates of model parameters and percentage misfits for inversions shown in Figure 4

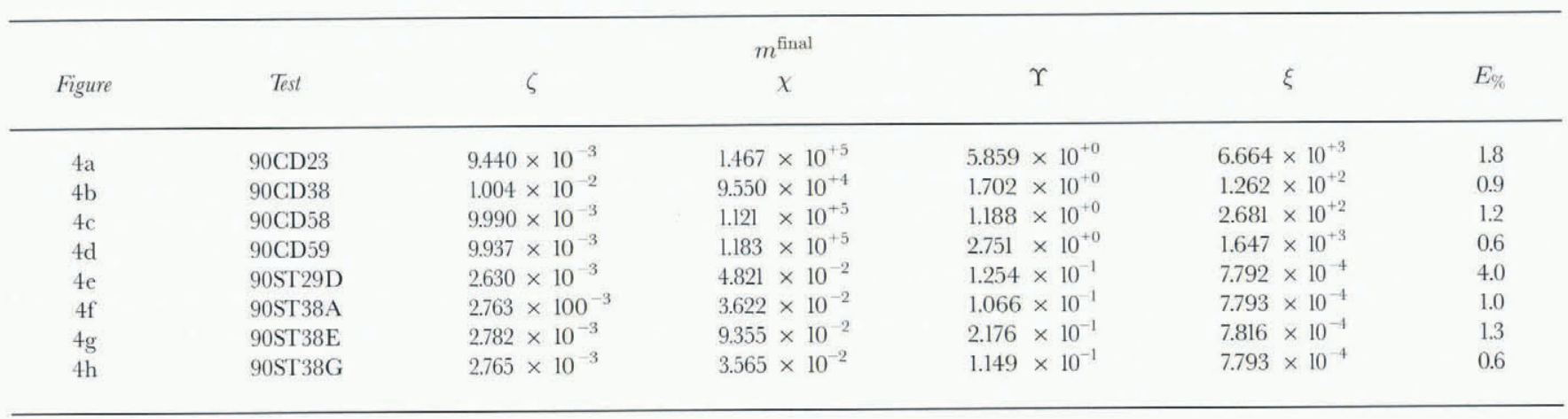

details are not perfectly matched (e.g. Fig. 4e), the basic behavior is still adequately expressed. As can be seen in Table 4, final parameter estimates from a particular type of response test are generally consistent. However, for some of the parameters, substantially different values were obtained from the two different types of test, even when they were performed in the same borehole. These results suggest that there are significant differences between connection-drainage and slug-test disturbances, either in the hydraulic properties to which a particular type of response test is sensitive or in the extent and kind of system that is sampled.

\section{DISGUSSION}

During a response test, friction between the borehole wall and the moving water column is accounted for by the skinfriction parameter $\zeta$. The inversion algorithm, which tests a wide range of parameter values, rendered estimates for $\zeta$ that are very small in magnitude and only slightly different from the initial estimates used to start the inversions (suggesting a lack of sensitivity to that parameter). Such small values for $\zeta$ indicate that wall-friction effects are insignificant during a response test. Likewise, final estimates of the Ergun parameter $\xi$ are negligibly small for slug tests, supporting the intuitive expectation that this type of disturbance is not associated with turbulent flow in the subglacial aquifer.

\section{Subglacial hydraulic properties}

Hydraulic properties of the subglacial flow layer, as it is characterized in the BHRT model, can be derived using Equations (5)-(8) and the estimated parameter values ob- tained from the inversions. The derived hydraulic properties are presented in Figure 5 and the basis for these results is discussed here.

Figure 5 a shows estimated values of hydraulic conductivity $K$ obtained from Equation (8b) and the connectiondrainage test values of $\xi$ listed in Table 4. (Because slug tests do not induce turbulent-flow conditions, estimates of $K$ based on slug-test values of $\xi$ are unreliable and thus will not be utilized.) To generate the estimates shown in Figure $5 \mathrm{a}$, we assigned plausible values to the other quantities that appear in Equation (8b). In porous media, a transition from laminar to turbulent flow occurs when $10 \leq \mathrm{Re} \leq 100$ (Marsily, 1986, p. 74). We assume that the Reynolds number at which this transition takes place is $\operatorname{Re}^{\prime}=55$. A likely range for porosity of the Trapridge Glacier substrate is $0.2 \leq n \leq 0.4$ (Clarke, 1987; Murray and Clarke, 1995). We assume that $n=0.3$. Based on a sedimentological size analysis of basal till from Trapridge Glacier, Clarke (1987) reported that the surface-to-volume ratio for fine-grained substrate material was $S_{0}=2.825 \times 10^{6} \mathrm{~m}^{-1}$. In this calculation, we assume that $S_{0}=3.0 \times 10^{6} \mathrm{~m}^{-1}$. The filter radius $r_{\mathrm{f}}$, which represents the excavation at the borehole base due to drilling, is assumed to be comparable to the radius of a typical borehole. Thus we take $r_{\mathrm{f}}=0.05 \mathrm{~m}$, as listed in Table 1 . With these assignments, the calculated hydraulic conductivities fall within the range $3-20 \times 10^{-3} \mathrm{~m} \mathrm{~s}^{-1}$, with a mean value of $1 \times 10^{-2} \mathrm{~m} \mathrm{~s}^{-1}$ (the dashed line in Figure $5 \mathrm{a}$ ). We point out that this method of calculating $K$, using Equation (8b) and drainage-test estimates of $\xi$, does not require any knowledge or assumption of the flow-layer thickness.

Intuitively, we expect that the flow layer is depleted of very fine-grained matrix material and mostly comprises sand- and gravel-sized particles, in which case the value of 

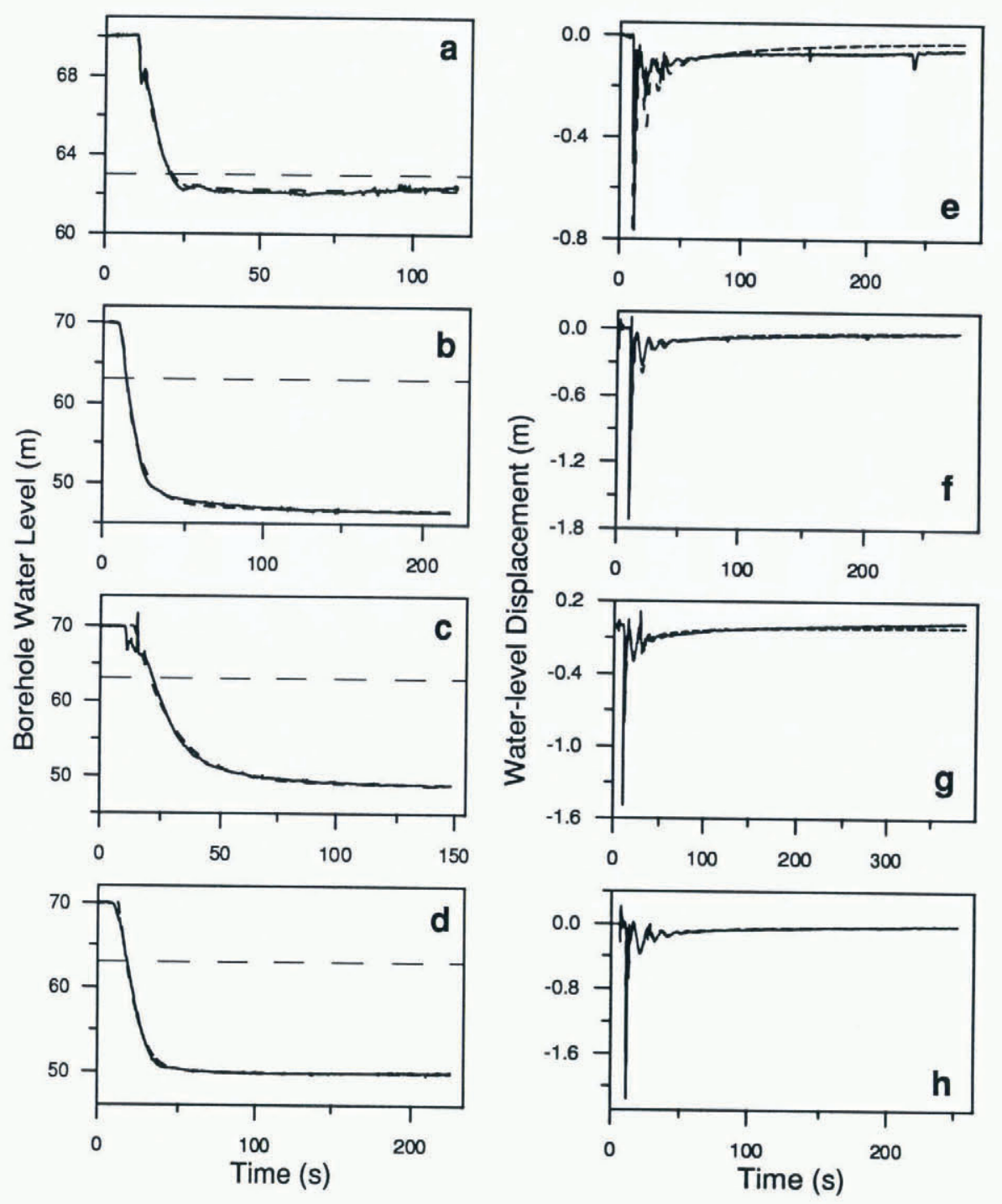

Fig. 4. Connection-drainage and slug-test data (solid lines) and inversion results (dashed lines). Drainage tests shown in the lefthand column are: (a) 90CD23; ( b) 90CD38; (c) 90CD58; (d) 90CD59. Horizontal dashed lines in the graphs in the lefthand column indicate the approximate ice-flotation level. Slug tests shown in the righthand column are: (e) 90ST29D; $(f)$ 90ST38A; (g) 90ST38E; (h) 90ST38G.

$S_{0}$ used in the previous calculations is inappropriate. (A typical diameter for coarse sand particles, say $1 \mathrm{~mm}$, corresponds to $S_{0}=6 \times 10^{3} \mathrm{~m}^{-1}$, three orders of magnitude smaller than the value assumed above.) To investigate the particle-size composition of the flow layer, we invoke the KozenyCarman relation

$$
k=\frac{n^{3}}{5 S_{0}^{2}(1-n)^{2}}
$$

(Carman, 1956), assuming that it applies in a subglacial environment, and combine it with the usual definition of hydraulic conductivity

$$
K=\frac{k \rho_{\mathrm{w}} g}{\eta}
$$

(e.g. Freeze and Cherry, 1979, p. 27) to obtain the following expression:

$$
K=\frac{\rho_{\mathrm{w}} g n^{3}}{5 \eta S_{0}{ }^{2}(1-n)^{2}}
$$

where $\rho_{\mathrm{w}}$ is the density of water, $g$ is the acceleration due to gravity and $\eta$ is the dynamic viscosity of water. For $n=0.3$ and $S_{0}=3 \times 10^{6} \mathrm{~m}^{-1}$, as assumed above, Equation (13) predicts that $K=6.7 \times 10^{-9} \mathrm{~m} \mathrm{~s}^{-1}$, significantly smaller than the results we obtained using Equation (8b) and drainage-test estimates of $\xi$. This inconsistency can be resolved by combining Equations (13) and (8b); after some algebraic manipulation, we obtain

$$
\frac{n(1-n)^{-\frac{2}{3}}}{S_{0}}=\left(\frac{5 \eta^{2} r_{\mathrm{f}} \operatorname{Re}^{\prime} \xi}{24 \rho_{\mathrm{w}}{ }^{2} g h_{0}}\right)^{\frac{1}{3}} .
$$

Now, for example, if we take $\xi=126.2$ (Table 4, 90CD38) and the other quantities on the righthand side of Equation (14) are as given above, then, for $n=0.2-0.4$, Equation (14) predicts that $S_{0}$ is in the range $2.9-7.1 \times 10^{3} \mathrm{~m}^{-1}$. This result does not depend on an assumed value for $K$ and it is consistent with the range of $S_{0}$ values expected for coarse-sand and fine-gravel sediment sizes. With this in mind, we again employ Equation (8b) to obtain estimates of hydraulic conductivity. However, this time we only consider values of $n$ and $S_{0}$ for which Equations (8b) and (13) are in good agreement in terms of predicting $K$. Using the results from $90 \mathrm{CD} 38(\xi=126.2)$ as an example, and spanning the range of plausible porosity values, we find that $K=1.6 \times 10^{-3} \mathrm{~m} \mathrm{~s}^{-1}$ for $n=0.2$ and $S_{0}=3 \times 10^{3} \mathrm{~m}^{-1}$ and $K=3.9 \times 10^{-3} \mathrm{~m} \mathrm{~s}^{-1}$ for $n=0.4$ and $S_{0}=7 \times 10^{3} \mathrm{~m}^{-1}$. In fact, for $n=0.35$ and $S_{0}=6 \times 10^{3} \mathrm{~m}^{-1}$, values of $\xi$ from the four drainage tests 

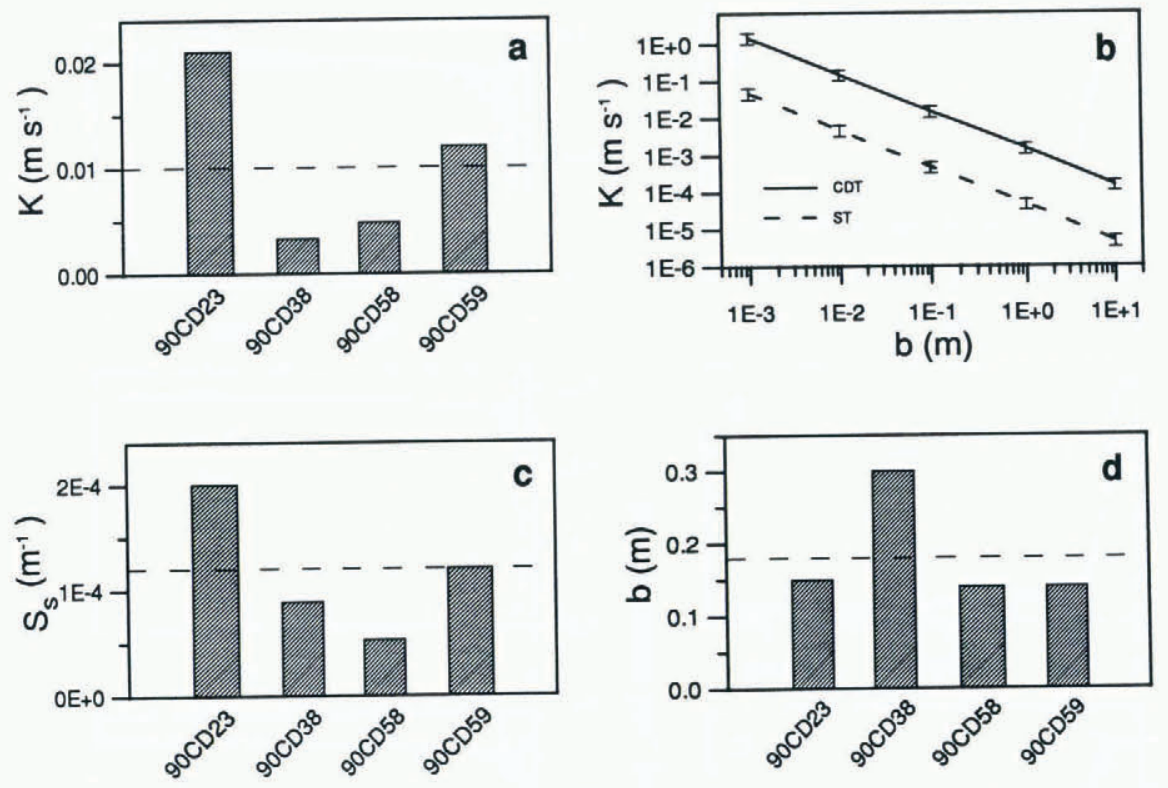

Fig. 5. Hydraulic properties derived from inversion results. Horizontal dashed lines indicate mean values. (a) Estimates of hydraulic conductivity obtained from connection -drainage test values of $\xi$. For reasons explained in the text, these estimates were subsequently reduced by a factor of about 20. (b) Transmissivity estimates obtained from final values of $\Upsilon$, broken down to show hydraulic conductivity as a function of flow-layer thickness for both connection-drainage tests (CDT) and slug tests (ST). Each line represents the mean value of results from four individual tests of a particular type. Vertical error bars indicate standard deviations of the means. (c) Estimates of specific storage obtained from connection-drainage test values of $\chi$ and $\Upsilon$. The actual value of specific storage is probably somewhat larger than these estimates, as discussed in the text. (d) Estimates of the flow-layer thickness obtained from connection-drainage test values of $\xi$ and $\Upsilon$.

(Table 4) again lead to hydraulic conductivities in the range $3^{-20} \times 10^{-3} \mathrm{~m} \mathrm{~s}^{-1}$, with a mean value of $1 \times 10^{-3} \mathrm{~m} \mathrm{~s}^{-1}$. (As a consistency check, we note that these values of $n$ and $S_{0}$ together with the Kozeny-Carman expression in Equation (13) predict $K=3.1 \times 10^{-3} \mathrm{~m} \mathrm{~s}^{-1}$.)

Transmissivity $T \equiv K b$ of the subglacial aquifer was extracted from Equation (7) using final estimates of the parameter $\Upsilon$ and an assumed value of $r_{\mathrm{w}}=0.05 \mathrm{~m}$ for the borehole radius. Transmissivity estimates have been broken down in Figure $5 \mathrm{~b}$ to show hydraulic conductivity as a function of flow-layer thickness for the two different types of response test. For each type of test, the corresponding line in Figure $5 b$ is the mean value of results from the four individual tests of that type, with vertical error bars showing the standard deviations of the means. The mean transmissivity for the connection-drainage tests is $1.52 \times 10^{-3} \mathrm{~m}^{2} \mathrm{~s}^{-1}$; for slug tests, the mean transmissivity is $7.75 \times 10^{-5} \mathrm{~m}^{2} \mathrm{~s}^{-1}$.

For a given aquifer thickness, hydraulic conductivity estimates derived from drainage tests are roughly an order-ofmagnitude larger than those estimated from slug tests (Fig. $5 b$ ). Differences in results from the two types of test are not due to spatial heterogeneity or poorly performed tests, as evidenced by the consistency of final parameter values for a particular type of test, even when both types of test were performed in the same borehole (e.g. hole 38 ). One possible explanation for this difference is that subglacial conditions are altered during connection-drainage tests, in which case the two types of test sample physically different systems; turbulent water flow during a drainage test could mobilize and flush fine-grained material from the flow layer in the vicinity of the borehole. However, in this situation one would expect subsequent tests to show an increase in hydraulic conductivity, rather than the decrease indicated by slugtest results.
Another possible explanation is that the overpressure condition that exists during the early stages of a connection-drainage test temporarily promotes ice-bed separation in the vicinity of the borehole, thereby facilitating water flow along the ice-bed interface, as well as through basal sediments. The following sequence of events satisfies physical intuition. A borehole is full of water until a connection is established. At the moment of connection, the pressure exerted on the glacier sole by water in the borehole exceeds the local ice-flotation pressure by about $11 \%$. The resulting glacier uplift provides an additional course for water exiting the borehole, effectively increasing the transmissivity for a brief period of time. During this time, a part of the increase in $T$ is due to expansion of the flow layer $b^{\prime}=b+\Delta b$, where $\Delta b$ is the height of the ice-bed separation. However, we expect that $\Delta b$ is small in comparison with $b$ (say $\sim 1 \%$ ), in which case the transient increase in $T$ is primarily due to an elevated "effective" hydraulic conductivity.

Acknowledging the effect of temporary ice-bed separation, Figure 5b suggests that estimates of hydraulic conductivity from drainage tests should be reduced by a factor of about 20 (the ratio of the mean transmissivity values given above), if they are to be representative of the subglacial flow layer under normal pressure conditions. Applying this correction to the estimated conductivities shown in Figure 5 a results in a revised mean value of $K=5 \times 10^{-4} \mathrm{~m} \mathrm{~s}^{-1}$, which we consider to be a valid estimate of the hydraulic conductivity of the basal-flow layer.

Figure 5c shows specific storage $S_{\mathrm{s}}$ of the subglacial flow layer derived using Equations (6) and (7) and the final estimates of $\chi$ and $\Upsilon$ from connection-drainage tests. Estimates of $S_{\mathrm{s}}$ from slug tests are notoriously unreliable, even to orders of magnitude (Papadopulos and others, 1973; Freeze 
and Cherry, 1979, p. 342; Marsily, 1986, p. 203; Harvey, 1992); thus, we exclude them from this discussion. Specific storage is obtained after dividing Equation (6) by Equation (7):

$$
S_{\mathrm{s}}=\frac{1}{2 b} \frac{\Upsilon}{\chi}\left(\frac{r_{\mathrm{w}}}{r_{\mathrm{f}}}\right)^{2} .
$$

To attain the results presented in Figure $5 c$, we have assumed that $b=0.1 \mathrm{~m}$ and $r_{\mathrm{w}}=r_{\mathrm{f}}$. With these assumptions, estimates of specific storage are in the range 5-20 $\times 10^{-5}$ $\mathrm{m}^{-1}$, with a mean value of $1.2 \times 10^{-4} \mathrm{~m}^{-1}$ (indicated by the dashed line in Figure 5c).

As a consequence of heterogeneity of hydraulic conditions at the glacier bed, we expect that specific storage of the overall glacier substrate may be somewhat underestimated by drainage-test results. To explain this point, consider the following: $S_{\mathrm{s}}$ can be described in words as the volume of water that an aquifer releases from storage per unit volume of aquifer per unit decline in hydraulic head, and it is defined mathematically as $S_{\mathrm{s}}=\rho_{\mathrm{w}} g(\alpha+n \beta)$, where $\alpha$ and $\beta$ are the compressibilities of the porous medium and water, respectively (Freeze and Cherry, 1979, p. 59); also, the compressibility of fine-grained sediments is typically greater than that of coarse-grained sediments, in some cases by several orders of magnitude (cf. Freeze and Cherry, 1979, p. 55). Hence, a porous medium comprising sand- and gravel-size particles will generally have less storage capacity than one comprising clay- and silt-size material.

In a region lacking a well-developed conduit or "canal" (cf. Walder and Fowler, 1994) system, subglacial water will tend to follow the least resistive paths through the substrate material or along its contact with basal ice. Parts of the substrate depleted of fine-grained matrix material offer less resistance to water flow, relative to those parts containing a greater percentage of silt- and clay-size particles, and likely form a network of preferential flow paths which constitute the subglacial drainage layer. Impulse disturbances, such as those imparted by connection-drainage and slug tests, affect only the relatively permeable zones within the substrate; longer-term stresses are required to obtain a response from the less-permeable regions bounding the flow zones. Hence, storage values inferred from drainage-test results are characteristic only of the fines-depleted flow zones and, on average, specific storage of the glacier substrate will be higher than that estimated from drainage tests, due to the greater percentage of fine-grained matrix material in regions that are not part of the active flow layer.

Estimates of the flow-layer thickness (Fig. 5d) can be obtained using the conductivity values shown in Figure $5 \mathrm{a}$ and the connection-drainage test relationship between $K$ and $b$ shown in Figure 5b, essentially combining results for the $\xi$ and $\Upsilon$ parameters and the assumptions that were discussed in connection with Figure $5 \mathrm{a}$ and b. (For connection- drainage tests, using revised estimates of $K$ and $T$ in this calculation is unnecessary, since both are corrected by an equal amount. Also, slug-test results cannot be used to estimate $b$ by this method because the parameter $\xi$ is insensitive to that type of test.) Estimates of the flow-layer thickness are in the range $0.1-0.3 \mathrm{~m}$, with a mean value of about $0.18 \mathrm{~m}$ (indicated by the dashed line in Figure $5 d$ ).

\section{Comparison with other studies}

Hydraulic properties of the substrate of Trapridge Glacier have been investigated by means other than response testing.
Murray and Clarke (1995) provided values for $K$ and (indirectly) $S_{\mathrm{s}}$ based on laboratory measurements of a basal till sample. They reported $K=2.2 \times 10^{-8} \mathrm{~m} \mathrm{~s}^{-1}$ and $(\alpha+n \beta)=$ $6.4 \times 10^{-6} \mathrm{~Pa}^{-1}$. As described above, the quantity $(\alpha+n \beta)$ must be multiplied by $\rho_{\mathrm{w}} g$ to obtain specific storage; for $\rho_{\mathrm{w}}=$ $1000 \mathrm{~kg} \mathrm{~m}^{-3}$ and $g=9.8 \mathrm{~m} \mathrm{~s}^{-2}$, this calculation yields $S_{\mathrm{s}}=$ $6.3 \times 10^{-2} \mathrm{~m}^{-1}$. In another study, Waddington and Clarke (1995) analyzed pressure fluctuations in boreholes that were not connected to the subglacial drainage system. They concluded that the hydraulic conductivity of the substrate in these regions was approximately $1-7 \times 10^{-9} \mathrm{~m} \mathrm{~s}^{-1}$ and reported compressibilities (p. 121; table 4) that correspond to values of specific storage in the range $\sim 10^{-4}$ to $10^{-1} \mathrm{~m}^{-1}$.

The values of hydraulic conductivity reported in both of these studies are substantially lower (several orders of magnitude) than our estimate of $K=5 \times 10^{-4} \mathrm{~m} \mathrm{~s}^{-1}$ for the basalflow layer. The difference is understandable for the following reasons: the study of Waddington and Clarke (1995) concerned the substrate at the base of boreholes that did not connect with the subglacial drainage system; the substrate in the vicinity of such boreholes must be significantly less permeable (conductive) than the drainage layer, since the substrate in these regions resists water flow, even in the presence of substantial hydraulic gradients. The laboratory results reported by Murray and Clarke (1995) were obtained from a sample of exposed basal till, which may not be representative of material comprising the drainage layer. Furthermore, as pointed out by Fountain (1994), laboratory experiments measure water flow only within the porous matrix and do not account for the possibility of additional routing along the ice-bed interface.

Our estimate of $S_{\mathrm{s}}$ lies at the low end of the range reported by Waddington and Clarke (1995), and is smaller than the value obtained by Murray and Clarke (1995). However, as explained above, the storage properties of the drainage layer are likely very different from those of the substrate in unconnected regions and the spatial mean value of $S_{\mathrm{s}}$ is probably underestimated by connectiondrainage test results.

In general, estimates of the hydraulic properties of the drainage system beneath Trapridge Glacier compare favorably with results reported from recent studies on other glaciers: based on analyses of average water levels and water-level variations in boreholes on South Cascade Glacier, Washington State, U.S.A., Fountain (1994) concluded that the hydraulic conductivity of a basal sediment layer of thickness $\sim 0.1 \mathrm{~m}$ was in the range $10^{-7}$ to $10^{-4} \mathrm{~m} \mathrm{~s}^{-1}$; Iken and others (1996) used an analytic solution to the standard radial diffusion equation (Marsily, 1986, p. 162), which applies to drainage from a borehole into a uniform sediment layer of fixed thickness $b$ and unlimited lateral extent, to analyze slug-test data from Gornergletscher, Valais, Switzerland. For $b=0.1 \mathrm{~m}$, their results suggest that $K=2 \times 10^{-2} \mathrm{~m} \mathrm{~s}^{-1}$, although they pointed out that this value may be too large due to the assumption of unlimited lateral extent of the sediment layer; Hubbard and others (1995) analyzed the propagation velocity of diurnal pressure waves to estimate the hydraulic diffusivity $\left(D=K / S_{\mathrm{s}}\right)$ of a sediment layer that participates in water movement at the bed of Haut Glacier d'Arolla, Valais, Switzerland. They reported diffusivities in the range $7 \times 10^{-2}$ to $4 \times 10^{1} \mathrm{~m}^{2} \mathrm{~s}^{-1}$. Our estimates of $K=$ $5 \times 10^{-4} \mathrm{~m} \mathrm{~s}^{-1}$ and $S_{\mathrm{s}}=1.2 \times 10^{-4} \mathrm{~m}^{-1}$ correspond to a value of $D=4.2 \times 10^{0} \mathrm{~m}^{2} \mathrm{~s}^{-1}$. 


\section{SUMMARY AND GONCLUDING REMARKS}

We have described an inverse approach for analyzing borehole response-test data and demonstrated use of the method with data collected on Trapridge Glacier. For response tests performed on glaciers, we have also shown that it is important to consider the background trend in basal water pressure during the course of the test as a fundamental part of the analysis procedure. Data from two different types of response test were examined; consistent differences between results from the two types of test stimulated further consideration of the subglacial processes associated with each type of test.

Inversions of data from both connection-drainage and slug tests resulted in a close match between the field observations and the inversion results, implying that the forward model characterization and final parameter estimates provide a reasonable hydrologic representation of the sub-glacial drainage system. Results of the inversions suggest that the subglacial drainage network of Trapridge Glacier can be described as a confined layer comprising coarsesand- to fine-gravel-sized sediments, having a thickness of $0.1-0.3 \mathrm{~m}$ and a hydraulic conductivity of about $5 \times 10^{-4} \mathrm{~m} \mathrm{~s}^{-1}$. Based on connection-drainage test results, specific storage of the flow layer was estimated to be roughly $1 \times 10^{-4} \mathrm{~m}^{-1}$.

Consideration of the physical effects of a borehole connection suggests that drainage-test results are susceptible to overestimation of hydraulic conductivity and probably underestimate mean specific storage of the glacier substrate. Drainage-test estimates of $K$ can be revised using transmissivity information derived from slug tests. However, this approach cannot be used to verify estimates of $S_{\mathrm{s}}$ because slug tests do not provide reliable information concerning specific storage.

\section{ACKNOWLEDGEMENTS}

This research was funded by the Natural Sciences and Engineering Research Council of Canada and by the U.S. National Science Foundation under grant DPP92-24244. We thank Parks Canada and the Yukon Territorial Government for permission to conduct research in Kluane National Park. Logistical support for the field program was provided by the Kluane Lake Research Station, operated by the Arctic Institute of North America. We are grateful to B. Barron and R. Grubin of the National Center for Atmospheric Research for computing assistance, and to M. Meier and A. Fountain for helpful discussions. Comments by A. Iken stimulated many improvements to the manuscript.

\section{REFERENCES}

Carman, P. C. 1956. Flow of gases through porous media. London, Butterworths Scientific.

Carrera, J. and S. P. Neuman. 1986. Estimation of aquifer parameters under transient and steady state conditions. 1. Maximum likelihood method incorporating prior information. Water Resour. Res., 22 (2), 199-210.

Clarke, G. K. C. 1987. Subglacial till: a physical framework for its properties and processes. 7. Geophys. Res., 92(B9), 9023-9036.

Cooper, H. H., Jr, J. D. Bredehoeft, I. S. Papadopulos and R. R. Bennett. 1965. The response of well-aquifer systems to seismic waves. 7 . Geophys. Res., 70 (16), 3915-3926.

Engelhardt, H. 1978. Water in glaciers: observations and theory of the behaviour of water levels in boreholes. Z. Gletscherkd. Glazialgeol., 14(1), $35-60$.
Engelhardt, H., N. Humphrey and B. Kamb. 1990. Borehole geophysical observations on Ice Stream B, Antarctica. Antarct. J. U.S., 25(5), 80-82.

Fountain, A. G. 1994. Borehole water-level variations and implications for the subglacial hydraulics of South Cascade Glacier, Washington State, U.S.A. J. Glaciol., 40 (135), 293-304.

Freeze, R. A. and J. A. Cherry. 1979. Groundwater. Engelwood Cliffs, NJ, Prentice-Hall.

Hairer, E. and G. Wanner. 1991. Solving ordinary differential equations II: stiffand differential-algebraic problems. Berlin, Springer-Verlag.

Harvey, C. F. 1992. What does a slug test measure in a heterogeneous aquifer? [Abstract.] EOS, 73(43), Supplement, 215.

Hodge, S. M. 1976. Direct measurement of basal water pressures: a pilot study. F. Glaciol., 16 (74), 205-218.

Hubbard, B. P., M. J. Sharp, I. C. Willis, M. K. Nielsen and C. C. Smart. 1995. Borehole water-level variations and the structure of the subglacial hydrological system of Haut Glacier d'Arolla, Valais, Switzerland. 7. Glaciol., 41 (139), 572-583.

Iken, A., K. Fabri and M. Funk. 1996. Water storage and subglacial drainage conditions inferred from borehole measurements on Gornergletscher, Valais, Switzerland. f. Glaciol., 42(141), 233- 248.

Kahaner, D., C. Moler and S. Nash. 1989. Numerical methods and software. Englewood Cliffs, NJ, Prentice-Hall.

Kamp, G. van der. 1976. Determining aquifer transmissivity by means of well response tests: the underdamped case. Water Resour. Res., 12(1), 71-77.

Kipp, K. L. 1985. Type curve analysis of inertial effects in the response of a well to a slug test. Water Resour. Res., 21 (9), 1397-1408.

Marsily, G. de. 1986. Quantitative hydrogeology. San Diego, CA, Academic Press Inc.

Menke, W. 1989. Geophysical data analysis: discrete inverse theory. Revised edition. New York, Academic Press. (International Geophysical Series 45.)

Murray, T. and G. K. C. Clarke. 1995. Black-box modeling of the subglacial water system. J. Geophys. Res., 100 (B7), 10,231-10,245.

Papadopulos, I. S., J. D. Bredehoeft and H. H. Cooper, Jr. 1973. On the analysis of slug test data. Water Resour. Res., 9(4), 1087-1089.

Press, W. H., B. P. Flannery, S. A. Teukolsky and W.T. Vetterling. 1989. Numerical recipes; the art of scientific computing (Fortran version). Cambridge, Cambridge University Press.

Stone, D. B. and G. K. C. Clarke. 1993. Estimation of subglacial hydraulic properties from induced changes in basal water pressure: a theoretical framework for borchole-response tests. 7. Glaciol., 39 (132), 327-340.

Tikhonov, A. N. and V.Y. Arsenin. 1977. Solutions of ill-posed problems. Washington, DC, V. H. Winston and Sons.

Waddington, B. S. and G. K. C. Clarke. 1995. Hydraulic properties of subglacial sediment determined from the mechanical response of waterfilled boreholes. f. Glaciol., 41 (137), 112-124.

Walder, J. S. and A. Fowler. 1994. Channelized subglacial drainage over a deformable bed. J. Glaciol., 40(134), 3-15.

Yeh, W.W. -G. 1986. Review of parameter identification procedures in groundwater hydrology: the inverse problem. Water Resour. Res., 22(2), 95-108.

\section{APPENDICES}

\section{A. Objective function}

The objective function provides a measure of the discrepancy between predicted and observed data. The method of least squares is commonly employed for this purpose. The objective function that we use (Equation (9)) is based on a least-squares approach involving two separate terms, which are described in this Appendix.

The idea behind the method of least squares is to find the set of model parameters that leads to the smallest total data misfit

$$
E=\sum_{i=1}^{N}\left(d_{i}^{\mathrm{obs}}-d_{i}\right)^{2}
$$

where $N$ is the number of data. Typically, the individual data-misfit terms are weighted according to the estimated uncertainties in the observed data $\delta d_{i}^{\text {obs }}$, and the total misfit is normalized by dividing by $N$. These modifications, applied to Equation (Al), lead to the following objective function: 


$$
\Phi_{\mathrm{d}}=\frac{1}{N} \sum_{i=1}^{N}\left(\frac{d_{i}^{\mathrm{obs}}-d_{i}}{\delta d_{i}^{\mathrm{obs}}}\right)^{2}
$$

The goal is to obtain the smallest value of $\Phi_{\mathrm{d}}$, since this minimizes the total misfit between predicted and observed data. However, for two reasons, it is necessary to modify Equation (A2) before we proceed. First, the differential equations that comprise the forward model are coupled, non-linear and stiff. The stiffness arises because diffusion terms in the PDE (2) are translated by divided differences into a large system of ODEs (Hairer and Wanner, 1991, p. 5). Under these conditions, numerical integration fails if model parameters are too unrealistic. Thus, the inversion algorithm must be restricted to searching a region of model space in which numerical solutions are both plausible and feasible. Secondly, the inversions need to be regularized. Regularization assures that small changes in the data will produce only small changes in the model parameter estimates, so that the solution remains stable (Tikhonov and Arsenin, 1977, p. 48). To guide and regularize inversions, we use a second objective function, which measures the parameter misfit:

$$
\Phi_{\mathrm{p}}=\frac{1}{M} \sum_{i=1}^{M}\left(\frac{m_{i}-m_{i}^{\mathrm{est}}}{\delta m_{i}^{\mathrm{est}}}\right)^{2}
$$

where $M$ is the number of parameters, $m_{i}^{\text {est }}$ are preliminary, "best-guess" parameter values and $\delta m_{i}^{\text {est }}$ are the uncertainties associated with each $m_{i}^{\text {est }}$. The final objective function

$$
\Phi=\Phi_{\mathrm{d}}+\lambda \Phi_{\mathrm{p}}
$$

includes a trade-off parameter $\lambda$, which is an adjustable constant that determines the relative importance of the parameter and data-misfit terms. Regularized, least-squares expressions like Equation (A4) are sometimes called "penalty functions" and are widely used in ground-water studies involving inversion for parameter identification (Carrera and Neuman, 1986; Yeh, 1986).

\section{B. Iterative-inversion procedure}

We start an inversion with a set of model parameters that is known from forward modeling to produce a reasonable fit to the observed data. At this stage, the estimated set of model parameters represents our best guess of what the final solution will be. When the first inversion is complete, the final set of model parameters will correspond to a minimum value of $\Phi$. We then start a second inversion by setting initial estimates equal to the final parameter values that were previously obtained. When the second inversion is complete, we compare the final value of $\Phi$ with that of the first inversion. In most cases, the two values of $\Phi$ agree to our specified level of precision. (Changes in $\Phi$ smaller than the precision that we specify - four significant digits correspond to parameter changes that do not appreciably alter the forward model solution.) In a few cases, however, additional inversions are required to reach consistent final values of $\Phi$; these situations arise when the parametermisfit term is significant. The end result of this iterative procedure is a "best-fitting" model, in the sense that the final objective-function value depends almost entirely on the data-misfit term.

Although the iterative procedure produces a best-fitting model, there is still uncertainty in the final result. Optimization methods based on Taylor expansions, such as the quasi-Newton method that we use, are guided by information on how a function behaves near a given point; they lack detailed information about a function's behavior over the entire region. These methods are sensitive to initial starting conditions, because the algorithm has only local information with which to search for a solution. Thus, the result of an inversion might be a local minimum of $\Phi$ instead of a global minimum. The algorithm that we use attempts to escape local minima by testing wide-ranging parameter values early in the inversion, then making more subtle changes as $\Phi$ becomes smaller; however, some uncertainty in the final solution is inevitable. Furthermore, there is also the possibility of non-uniqueness, as two or more solutions might produce equal-valued minima in $\Phi$. Uncertainty and non-uniqueness are intrinsic to many inverse problems and, in general, require some compromise in extracting information from any data set (Menke, 1989, p. 17). Monte Carlo or uniform grid-search techniques could be used to help locate a global minimum but these procedures would be impractical for inversions of multiple-response tests - the computational requirements of performing even a few thousand inversions of a single-data set are prohibitive. In the light of these considerations, we use the phrase "estimates of model parameters" when referring to an inverse solution. 